\title{
Costos económicos de neumonía adquirida en comunidad, meningitis y bacteriemia por Streptococcus pneumoniae en una población adulta que requirió hospitalización en Bogotá, Colombia
}

\author{
Claudia Calderón ${ }^{1}$, Rodolfo Dennis² \\ 1 Investigadora independiente, Bogotá, D.C., Colombia \\ 2 Departamento de Investigaciones, Fundación Cardioinfantil-Instituto de Cardiología, Bogotá, D.C., Colombia
}

Introducción. Las infecciones por Streptococcus pneumoniae en adultos se relacionan con neumonía, meningitis y bacteriemia. El costo de la atención en personas adultas no está bien documentado en Colombia, pero puede ser significativo.

Objetivo. Analizar los costos asociados con la neumonía adquirida en comunidad, meningitis y bacteriemia por $S$. pneumoniae en adultos hospitalizados en Colombia y estimar el costo de la atención ambulatoria de esta neumonía. Servir de base para futuras evaluaciones económicas.

Materiales y métodos. Se realizó un estudio de costos directos asociados a neumonía adquirida en comunidad, meningitis y bacteriemia por $S$. pneumoniae confirmado por cultivos. Se tomó una muestra de conveniencia de adultos hospitalizados entre enero de 2010 y junio de 2011 en tres hospitales de tercer nivel de Bogotá. Se analizaron 107 registros y se obtuvieron 60 facturas cobradas al pagador. La información se clasificó por costos de atención y tratamiento. Para los casos ambulatorios de neumonía, el estimativo de costos se hizo utilizando metodología Delphi con expertos clínicos.

Resultados. El promedio de los costos directos totales asociados con neumonía fue de Col\$12'178.949, Col\$ 7’533.187 para meningitis y Col\$ 9’242.806 para bacteriemia. La neumonía se presentó en 70 $\%$ de los hombres y $30 \%$ de las mujeres; la meningitis se distribuyó igual en ambos sexos (50 \%) y la bacteriemia se presentó en $67 \%$ de los hombres y $33 \%$ de las mujeres. El costo por adulto de la atención ambulatoria de la neumonía adquirida en la comunidad se estimó en Col\$106.174. Para casos especiales se incrementó a Col\$164.695.

Conclusión. La enfermedad neumocócica en adultos, especialmente mayores de 45 años, representa un alto costo por el empleo de medicamentos y la estancia hospitalaria, que causan un impacto en los recursos del sistema. La prevención y el tratamiento temprano de las neumonías pueden disminuir costos y reducir la carga de enfermedad.

Palabras clave: costos de la atención en salud, neumonía, meningitis, bacteriemia, Streptococcus pneumoniae, costo de enfermedad, economía, costos y análisis de costo, Colombia.

doi: http://dx.doi.org/10.7705/biomedica.v34i1.1553

Economic cost of Streptococcus pneumoniae community-acquired pneumonia, meningitis and bacteremia in an adult population that required hospitalization in Bogotá, Colombia

Introduction: Streptococcus pneumoniae infection in adults is related to pneumonia, meningitis and bacteremia. Its care costs in adults are not well documented in Colombia and it has a greater impact in people over 45 years old.

Objectives: The aims of this study were to analyze the associated costs of pneumonia, bacteremia and meningitis in invasive $S$. pneumoniae infection in Colombia among hospitalized adults and to estimate outpatient costs for community-acquired pneumonia. Additionally, we wanted to serve as a starting point for future economic evaluations.

Materials and methods: We performed a direct cost study associated with S. pneumoniae outpatient community-acquired pneumonia, bacteremia and meningitis costs confirmed by cultures. A cohort of hospitalized adults treated between January 2010 and June 2011 in three third level hospitals in Bogotá was analyzed. We evaluated 107 records and 60 bills charged to the payer. The data were classified according to care and treatment costs. We performed an estimate of direct costs for community-acquired pneumonia for outpatient cases through Delphi methodology using expert clinicians.

\section{Contribución de los autores:}

Claudia Calderón: participación en la planeación del estudio, recolección de datos, organización de la base de datos y análisis de resultados. Rodolfo Dennis: participación en la planeación del estudio, interpretación de resultados y realización del panel de expertos con metodología Delphi.

Los dos autores participaron en la redacción, revisión y corrección del manuscrito. 
Results: The average direct costs associated with pneumococcal disease were US $\$ 6,283$, US $\$ 3,886$, and US $\$ 4,768$ for pneumonia, meningitis and bacteremia, respectively (exchange rate 1 US $\$=$ Col $\$$ 1,938.34; average variation between 2010 and 2011). Pneumonia cases were 70\% men and $30 \%$ women; the distribution for meningitis was the same for both genders (50\%); and for bacteremia we had $67 \%$ men and $33 \%$ women. Outpatient cost of community-acquired pneumonia was estimated at US\$ 82.2 (Col\$159,280) in adults. For special cases, direct cost increased to US\$ 142 (Col\$274,427).

Conclusions: The management of $S$. pneumoniae infection in people over 45 years old represents a high cost due to the use of drugs and hospitalization, which has a direct impact on health resources. Prevention and early treatment for pneumonia can reduce the cost and the burden of the disease.

Keywords: health care costs, pneumonia, meningitis, bacteremia, Streptococcus pneumoniae, cost of illness, economics, costs, cost analysis, Colombia.

doi: http://dx.doi.org/10.7705/biomedica.v34i1.1553

La Organización Mundial de la Salud (OMS) estima que las neumonías por neumococo en adultos en países industrializados representa, al menos, $30 \%$ de todos los pacientes hospitalizados con neumonía adquirida en la comunidad, con una tasa de mortalidad de 11 a $44 \%$. En el 2002 la incidencia anual de neumonía adquirida en comunidad en los Estados Unidos fue de 18,3 casos por 100.000 habitantes adultos y la incidencia de neumonía neumocócica en la población anciana fue de cerca de 5,5 casos por 100.000 habitantes (1).

La enfermedad respiratoria causa 1,6 millones de muertes en el mundo; en Latinoamérica se estima que hay 3.637 casos anuales de meningitis, 14.548 de bacteriemia, de 192.761 a 318.238 casos de neumonía y cerca de 5,6 millones de casos de otitis media al año (2).

De igual forma, se calcula que $6 \%$ de los fallecimientos se deben a infecciones respiratorias bajas, y Streptococcus pneumoniae es el agente causal de $35 \%$ de estos casos (3). En los estudios económicos, el impacto de la enfermedad se ha medido con base en costos generales, pero rara vez en los costos reales (4).

La ausencia de datos de costos directos locales en la enfermedad por $S$. pneumoniae en adultos es un factor que ha resultado en la subestimación del beneficio de la vacunación y de la expansión de los programas de salud; el sistema de salud debería contemplar la posibilidad de establecer nuevas políticas orientadas a proteger grupos vulnerables, como la población mayor de 65 años, solamente en términos de costos (5).

\footnotetext{
Correspondencia:

Claudia Calderón, Departamento de Investigaciones, Fundación Cardioinfantil-Instituto de Cardiología, Carrera 13B № 161-85, torre H, piso 3, Bogotá, D.C., Colombia

Teléfono: (571) 667 2727, ext. 6301

claudiapcalderon@hotmail.com
}

Recibido: 22/01/13; aceptado: 28/09/13
El conocimiento de la verdadera incidencia de la enfermedad neumocócica en adultos mayores es limitado debido a las dificultades del levantamiento del costo y del aislamiento del microorganismo específico en los sujetos sintomáticos respiratorios. El riesgo de enfermar por neumonía o meningitis está más relacionado con edades extremas, niños menores de cinco años y adultos mayores de 65 años, que con la prevalencia de enfermedades concomitantes tales como las enfermedades cardiovasculares, pulmonares y hepáticas crónicas que complican el desenlace porque incrementan el riesgo de infección e incluso la mortalidad (6). Los estudios realizados en adultos mayores de 60 años en Bogotá se basan en los costos aproximados de la enfermedad por S. pneumoniae. En el 2008 se presentaron 6.585 neumonías, 689 de ellas por $S$. pneumoniae y 73 casos de meningitis, 12 de ellos $(16,4 \%)$ por el mismo agente (3).

Para el estudio de costos de neumonía, meningitis y bacteriemia se requiere, además del costo total de una enfermedad o de una intervención en salud, la discriminación de los componentes del costo. Esto hace referencia a qué proporción del costo representan los medicamentos, la estancia hospitalaria, las consultas médicas, las pruebas de laboratorio, los exámenes de apoyo diagnóstico y las terapias. Este análisis de los determinantes del costo es importante no sólo para entender qué factores inciden en él, sino por su relevancia en el momento en que un estudio económico se adapte a otras circunstancias en la que los costos unitarios sean diferentes (4).

El objetivo de este estudio fue obtener información local específica sobre los costos directos con base en una muestra de pacientes hospitalizados con neumonía, meningitis y bacteriemia por S. pneumoniae. Se incluyeron los costos por hospitalización en la unidad de cuidados intensivos para los casos de neumonía, meningitis y bacte- 
riemia que requirieron soporte respiratorio. El cálculo de los costos de neumonía adquirida en la comunidad se realizó con la metodología Delphi a partir de casos ambulatorios.

\section{Materiales y métodos}

Este es un estudio descriptivo basado en una revisión y análisis estadístico de los costos directos de la neumonía adquirida en la comunidad, la meningitis y la bacteriemia por $S$. pneumoniae en sujetos mayores de 18 años, hospitalizados en tres hospitales de tercer nivel de Bogotá, entre enero de 2010 y junio de 2011. Se evaluaron los datos sobre los costos de 107 registros de sujetos con cultivos positivos para $S$. pneumoniae y se analizaron los costos de 60 facturas cobradas al pagador que cumplieron con criterios de inclusión del estudio. Los costos analizados en este estudio fueron costos directos desde la perspectiva del pagador y los datos se desglosaron y analizaron con base en las órdenes pagadas a las empresas promotoras de salud (EPS) del régimen contributivo y subsidiado que hubieran sido revisadas y auditadas.

El alcance del estudio se fijó para sujetos hospitalizados. Los medicamentos, controles o procedimientos posteriores al momento del alta no se tuvieron en cuenta.

Se incluyeron los tres primeros diagnósticos de egreso que estuvieran relacionados con el código de la clasificación internacional de enfermedades (CIE-10), en este caso, neumonías bacterianas no especificadas y especificadas, bronconeumonías, neumonía lobar y derrame pleural. En el caso de la meningitis se tuvieron en cuenta la meningitis estreptocócica y la meningitis neumocócica y en el de la bacteriemia, la septicemia estreptocócica, la septicemia debida a $S$. pneumoniae, la septicemia estreptocócica no especificada y la infección estreptocócica sin otra especificación.

Se excluyeron los casos de tuberculosis activa, absceso pulmonar, neumonías hospitalarias, nuevas hospitalizaciones por neumonía, hospitalizaciones durante los 15 días anteriores, así como la quimioterapia por cáncer. No se incluyeron el trauma encefálico, los procedimientos neuroquirúrgicos, la meningitis por tuberculosis, nueva hospitalización por meningitis, la meningitis hospitalaria y la meningitis por hongos documentada. En todos los casos se excluyeron los sujetos con trasplante renal o coronario que estuvieran en tratamiento de inmunosupresión, los sujetos en tratamiento crónico con corticoides o en terapias biológicas.
La recolección de datos del estudio se hizo con el programa Excel de Microsoft y para el análisis estadístico se utilizó el programa Stata Corp., 2007. Stata Statistical Software: Release 10 (CollegeStation, TX: StataCorp LP). La información se organizó por grupos demográficos, por enfermedad asociada al perfil de riesgo y por costos de enfermedad. Los resultados de los costos se expresaron en promedio y desviación estándar.

La muestra se organizó bajo parámetros de indicadores de ocurrencia para los grupos de edad de 18 a 44 años, 45 a 64 años, 65 a 79 años y mayores de 80 años. Los perfiles de riesgo se organizaron en tres categorías: perfil bajo, inmunocompetente sin condiciones médicas crónicas; moderado, inmunocompetente con una o más condiciones médicas crónicas (enfermedad cardiovascular, hepática, pulmonar, renal o diabetes), y alto, sujeto inmunocomprometido ( $\mathrm{VIH}$, sida).

En la categorización de las variables de costos se incluyó la estancia con hospitalización en piso, urgencias y unidad de cuidados intensivos. Otra variable fue la de medicamentos, los cuales se agruparon según características terapéuticas en: antibióticos, inotrópicos, broncodilatadores, analgésicos, oxígeno, líquidos intravenosos y "otros". La variable de laboratorio clínico incluyó cuadro hemático, creatinina, nitrógeno ureico, cultivos (cultivo de esputo, tinción de Gram, hemocultivos, cultivo de líquido cefalorraquídeo), antibiograma, electrolitos, gases arteriales $y$ "otros". La de ayudas diagnósticas incluyó rayos X, TC de tórax, TC cerebral, resonancia magnética, ecografía, al igual que resultados de patología y "otros". Entre los procedimientos se contempló la punción lumbar, la broncoscopia, la toracoscopia, los derechos de cirugía e instrumentación, las intubaciones y las traqueotomías; los demás se agruparon bajo "otros". También se calcularon los costos de las interconsultas de terapia respiratoria, física, fonoaudiología y "otras".

El cálculo de los costos asociados con el manejo ambulatorio de la neumonía adquirida en la comunidad, se realizó con la metodología Delphi y mediante el consenso de tres expertos neumólogos. Los resultados fueron sometidos a una segunda ronda para llegar a un acuerdo sobre los componentes de los recursos de consulta externa o consulta de urgencias en pacientes con sospecha clínica de neumonía adquirida en la comunidad que no requieren hospitalización. Se tomaron las tarifas contempladas en el manual 
tarifario del Instituto de Seguros Sociales para 2001 más 30\% como caso de referencia, ya que tenían mayor representatividad en el mercado y se expresaron en pesos colombianos de 2010; la perspectiva utilizada para el análisis de costos corresponde a la de la EPS.

\section{Consideraciones éticas}

El estudio contó con la aprobación de los comités de ética de las tres instituciones.

\section{Resultados}

De 107 casos de neumonía, meningitis y bacteriemia confirmados, se evaluaron de forma retrospectiva las historias clínicas y las facturas de 60 sujetos que cumplían con los criterios de inclusión y cuyos cultivos habían sido positivos para $S$. pneumoniae en las tres entidades. De estos 60 casos, 40 correspondieron a hombres $(67 \%)$ y $20(33 \%)$ a mujeres.

\section{Neumonía adquirida en la comunidad}

Se presentaron 33 casos de neumonía adquirida en la comunidad, y se observó una mayor frecuencia de neumonía en el grupo de hombres (70\%) que en el de mujeres (30\%). La mediana de edad para neumonía fue de 62 años ( $\mathrm{DE}=18,2)$; en hombres la mediana fue de 67 años $(D E=19,8)$ y en mujeres, de 54,5 años ( $D E=14,3)$. Basados en el perfil de riesgo, el perfil moderado con mayores enfermedades concomitantes se presentó en $76 \%$ de los casos y con mayor frecuencia en el grupo de edad de 45 a 64 años (cuadro 1), lo cual se correlaciona con lo descrito por Sanguinetti, et al., en cuyo estudio la mediana de edad en personas con neumonía fue de 58 años y $64,5 \%$ del total de pacientes tenía un perfil de riesgo moderado (6). En el perfil de riesgo alto se presentaron $37,5 \%$ casos de infección por el virus de la inmunodeficiencia humana $(\mathrm{VIH})$ con mayor compromiso en el grupo de menor edad (18 a 44 años); no se presentaron casos de VIH en los grupos de más de 65 años. Las características generales de la población de estudio y su distribución según el tipo de afiliación al Sistema General de Seguridad en Salud (SGSSS) se describen en el cuadro 1.

\section{Variables de costos en neumonía adquirida en la comunidad}

Al determinar los costos directos totales para neumonía se halló un costo promedio de Col\$ 12'178.949 (DE=Col\$14'264.174). Para el análisis del tiempo de estancia se tuvieron en cuenta tres servicios de hospitalización diferentes, una
Cuadro 1. Características generales de los sujetos de la muestra y características sociodemográficas y de perfil de riesgo de la población analizada.

\begin{tabular}{lrrrrrr}
\hline & \multicolumn{2}{c}{ Neumonía } & \multicolumn{2}{c}{ Meningitis } & \multicolumn{2}{c}{ Bacteriemia } \\
\hline Variable & $\mathbf{n}$ & $\%$ & $\mathbf{n}$ & $\%$ & $\mathbf{n}$ & $\%$ \\
\hline Sexo & & & & & & \\
$\quad$ Masculino & 23 & 70 & 3 & 50 & 14 & 67 \\
$\quad$ Femenino & 10 & 30 & 3 & 50 & 7 & 33 \\
Edad (años) & & & & & & \\
$\quad 18$ a 44 & 7 & 21 & 5 & 83 & 4 & 20 \\
$\quad 45$ a 64 & 10 & 30 & 1 & 17 & 8 & 38 \\
$\quad 65$ a 79 & 9 & 28 & 0 & 0 & 3 & 14 \\
$\quad>0$ & 7 & 21 & 0 & 0 & 6 & 28 \\
Perfil de riesgo & & & & & & \\
$\quad$ Bajo & 3 & 9 & 3 & 50 & 3 & 14 \\
$\quad$ Moderado & 22 & 67 & 3 & 50 & 18 & 86 \\
$\quad$ Alto & 8 & 24 & 0 & 0 & 0 & 0 \\
Régimen de afiliación & & & & & & \\
$\quad$ Subsidiado & 11 & 33 & 3 & 50 & 9 & 43 \\
$\quad$ Contributivo & 22 & 67 & 3 & 50 & 12 & 57 \\
$\quad$ Mortalidad & & & & & & \\
$\quad$ Sí & 7 & 21 & 2 & 33 & 10 & 48 \\
$\quad$ No & 26 & 79 & 4 & 67 & 11 & 52 \\
\hline
\end{tabular}

hospitalización en piso, otra en unidad de cuidados intensivos y otra en urgencias. Se encontró que el costo promedio de la estancia fue de Col\$ 4'246.754 (DE=Col\$ 5'954.456); $51 \%$ de este valor correspondió a la estancia en la unidad de cuidados intensivos, $35 \%$ a la hospitalización en piso y $14 \%$ a urgencias. El análisis del costo según el perfil de riesgo mostró que los sujetos inmunocompetentes de perfil bajo tuvieron la menor estancia hospitalaria (mediana de 6 días); los sujetos con perfil moderado con una o más enfermedades concomitantes (hipertensión arterial, enfermedad cardiovascular, diabetes) (7) presentaron una mediana de estancia de 15 días mientras que la de los sujetos con perfil de riesgo alto con inmunocompromiso fue de 17 días.

Al valorar el comportamiento de las variables de los costos directos se encontró que las que las de mayor costo fueron la estancia y el consumo de medicamentos. En el estudio de Reyes, et al., 69,3 $\%$ de los costos totales por neumonía correspondió a la estancia, mientras que en nuestro estudio, el $44 \%$ del costo total correspondió a medicamentos (7). El costo de estancia para neumonía fue de Col\$ 4'246.754 (DE=Col\$ 5'954.456) y el de medicamentos fue de Col\$5'309.214 (DE= Col\$ 5'940.326), lo que representó 35 y $44 \%$ del costo total, respectivamente, seguidos de las ayudas diagnósticas con $7 \%$ del costo, es decir, Col\$ 888.832 ( $\mathrm{DE}=\mathrm{Col} \$ 1$ '016.599) y de las pruebas de laboratorio con $7 \%$ del costo total (Col\$ 869.044, 
$\mathrm{DE}=\mathrm{Co} \$$ 1'071.991) (figura 1). El menor aporte al costo en neumonía fue por interconsultas (Col\$ $664.773, \mathrm{DE}=\mathrm{Col} \$ 795.433$ ) y por procedimientos (Col\$ 200.331, DE=Col\$ 553.148) (cuadro 2).

Al discriminar los costos de los medicamentos para el tratamiento de la neumonía, se observó que $36 \%$ correspondía a los antibióticos, lo que se relacionó directamente con el tratamiento de elección para combatir la infección causada por $S$. pneumoniae (figura 2).

\section{Meningitis}

Se presentaron seis casos de meningitis con igual distribución en hombres y en mujeres (50\%). La mediana de edad fue de 33 años ( $D E=11,3)$, de 30 años en hombres $(\mathrm{DE}=6,8)$ y de 34 años en mujeres $(D E=14,8)$. El tiempo de estancia presentó una mediana de 11 días $(D E=8,13)$ en unidad de cuidados intensivos y de 4,5 días de estancia en el caso de los fallecidos. En el grupo de 18 a 44 años se presentó $83 \%$ de los casos de meningitis y el 17 $\%$ restante, en el grupo de 45 a 64 años. La variable de costos para meningitis fue de Col\$ 7'533.187 ( $\mathrm{DE}=$ Col $\left.\$ 66^{\prime} 420.528\right)$, y la variable de mayor peso en el costo del manejo de la meningitis fue la estancia (Col\$ 3'546.990, DE=Col\$ 3'221.352) con $47 \%$ del costo. En la meningitis, la variable de medicamentos correspondió a $23 \%$ del costo total y el grupo de "otros" medicamentos representó $39 \%$ del costo total de medicamentos tales como sedantes, heparinas de bajo peso molecular y relajantes musculares, entre otros, seguidos por los antibióticos con $33 \%$, oxígeno con 14,5\%, líquidos intravenosos con $8 \%$ e inotrópicos con $5 \%$ (figura 2).

\section{Bacteriemia}

Se presentaron 21 casos de bacteriemia, $67 \%$ en hombres y $33 \%$ en mujeres. La mediana de edad para hombres fue de 60,5 años $(D E=20,4)$ y para mujeres, de 60 años $(D E=16,5)$. En cuanto a los rangos de edad se observó que $38 \%$ de los casos se presentaron en el grupo de 45 a 64 años y $28 \%$ en el de mayores de 80 años. El tiempo de estancia presentó una mediana de seis días $(D E=12,3)$ en la unidad de cuidados intensivos y de cuatro días en los casos de fallecidos.

La variable de costos promedio para bacteriemia fue de Col\$ 9'242.806 (DE=Col\$2'625.596). Al valorar el comportamiento de las variables para costos directos, se encontró que las de mayor costo fueron los medicamentos Col\$ 5'598.678 (DE=Col $\$ 713.907)$ con $61 \%$ del coto total, y la de la estancia con Col\$2'329.501 (DE=Col\$ 1'615.932), es decir, $25 \%$ (cuadro 2).

En general, los medicamentos que más incidieron sobre el costo total fueron los antibióticos con $39,4 \%$, seguidos por el grupo de medicamentos clasificado como "otros" con $43 \%$, el oxígeno con $6,4 \%$, los líquidos intravenosos con $4,5 \%$ y los medicamentos inotrópicos con $5 \%$ tuvieron un menor impacto sobre el costo total. En la bacteriemia se observaron variaciones, ya que el grupo de antibióticos tiene una mayor participación

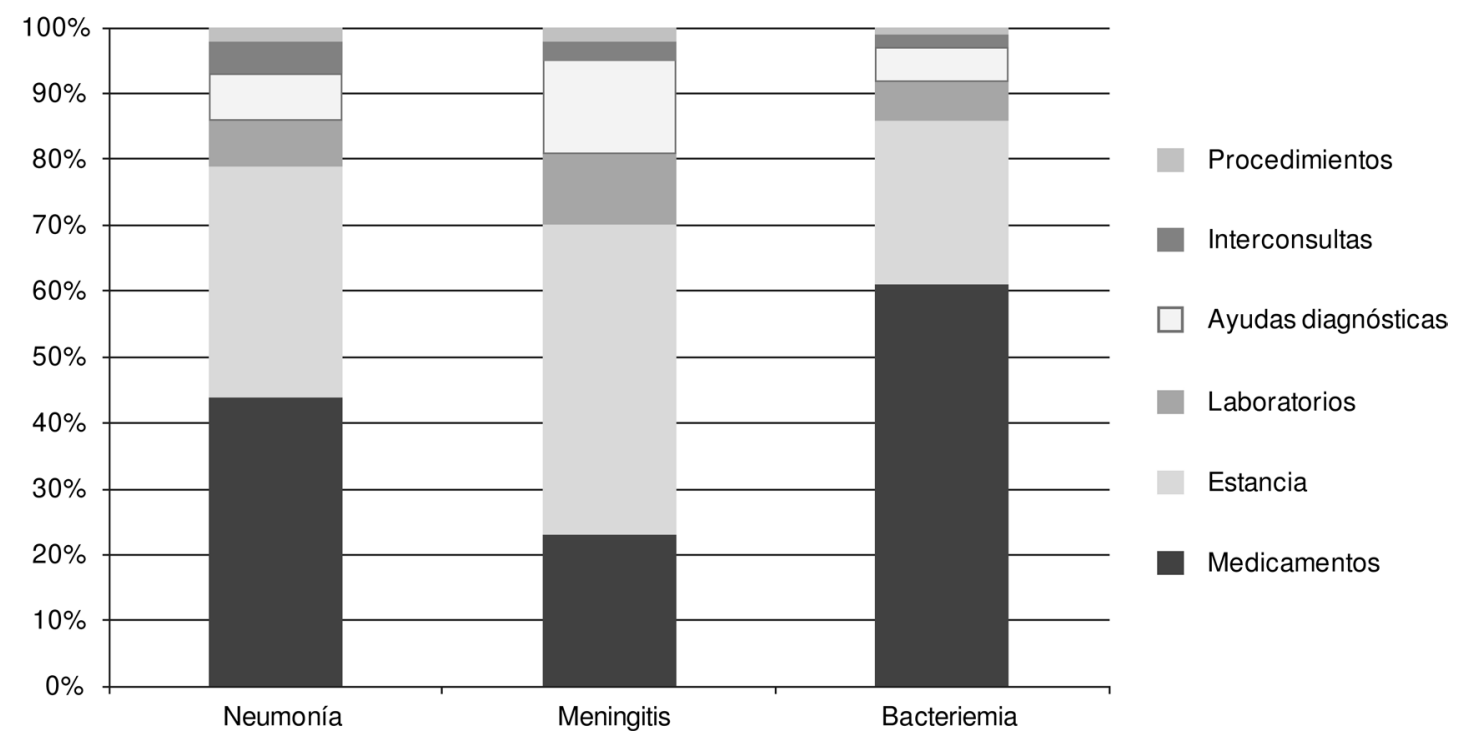

Figura 1. Variables de distribución de costos directos según patología (porcentaje). 
Cuadro 2. Costos promedio de neumonía, meningitis y bacteriemia por Streptococcus pneumoniae (desviación estándar)

\begin{tabular}{|c|c|c|c|c|c|c|}
\hline \multirow[b]{2}{*}{ Variable } & \multicolumn{2}{|l|}{ Neumonía } & \multicolumn{2}{|l|}{ Meningitis } & \multicolumn{2}{|c|}{ Bacteriemia } \\
\hline & Pesos & $\%$ & Pesos & $\%$ & Pesos & $\%$ \\
\hline Medicamentos & $\begin{array}{r}\$ 5,309.214 \\
\left(\mathrm{DE}=\$ 5^{\prime} 940.326\right)\end{array}$ & 44 & $\begin{array}{r}\$ 1 ' 755.180 \\
\left(\mathrm{DE}=\$ 1^{\prime} 447.482\right)\end{array}$ & 23 & $\begin{array}{r}\$ 5 ’ 598.678 \\
(\mathrm{DE}=\$ 713.907)\end{array}$ & 61 \\
\hline Estancia & $\begin{array}{r}\$ 4^{\prime} 246.754 \\
\left(\mathrm{DE}=\$ 5^{\prime} 954.456\right)\end{array}$ & 35 & $\begin{array}{r}\$ 3^{\prime} 546.990 \\
\left(\mathrm{DE}=\$ 3^{\prime} 221.352\right)\end{array}$ & 47 & $\begin{array}{r}\$ 2 ' 329.501 \\
\left(\mathrm{DE}=\$ 1^{\prime} 615.932\right)\end{array}$ & 25 \\
\hline Laboratorios & $\begin{array}{r}\$ 869.044 \\
\left(\mathrm{DE}=\$ 1^{\prime} 071.991\right)\end{array}$ & 7 & $\begin{array}{r}\$ 805.864 \\
(\mathrm{DE}=\$ 718.347)\end{array}$ & 11 & $\begin{array}{r}\$ 532.498 \\
(\mathrm{DE}=\$ 370.982)\end{array}$ & 6 \\
\hline Ayudas diagnósticas & $\begin{array}{r}\$ 888.832 \\
\left(\mathrm{DE}=\$ 1^{\prime} 016.599\right)\end{array}$ & 7 & $\begin{array}{r}\$ 1 \text { 1'063.606 } \\
\left(\mathrm{DE}=\$ 1^{\prime} 420.385\right)\end{array}$ & 14 & $\begin{array}{r}\$ 508.131 \\
(\mathrm{DE}=\$ 370.030)\end{array}$ & 5 \\
\hline Interconsultas & $\begin{array}{r}\$ 664.773 \\
(\mathrm{DE}=\$ 795.433)\end{array}$ & 5 & $\begin{array}{r}\$ 242.284 \\
(D E=\$ 208.266)\end{array}$ & 3 & $\begin{array}{r}\$ 172.763 \\
(D E=\$ 216.785)\end{array}$ & 2 \\
\hline Procedimientos & $\begin{array}{r}\$ 200.331 \\
(\mathrm{DE}=\$ 553.148)\end{array}$ & 1,6 & $\begin{array}{r}\$ 119.263 \\
(\mathrm{DE}=\$ 184.163)\end{array}$ & 2 & $\begin{array}{r}\$ 101.235 \\
(\mathrm{DE}=\$ 247.974)\end{array}$ & 1 \\
\hline Costo total & $\begin{array}{r}\$ 12^{\prime} 178.949 \\
\left(\mathrm{DE}=\$ 14^{\prime} 264.174\right)\end{array}$ & 100 & $\begin{array}{r}\$ 7^{\prime} 533.187 \\
\left(\mathrm{DE}=\$ 6^{\prime} 420.528\right)\end{array}$ & 100 & $\begin{array}{r}\$ 9^{\prime} 242.806 \\
\left(\mathrm{DE}=\$ 2^{\prime} 625.596\right)\end{array}$ & 100 \\
\hline
\end{tabular}

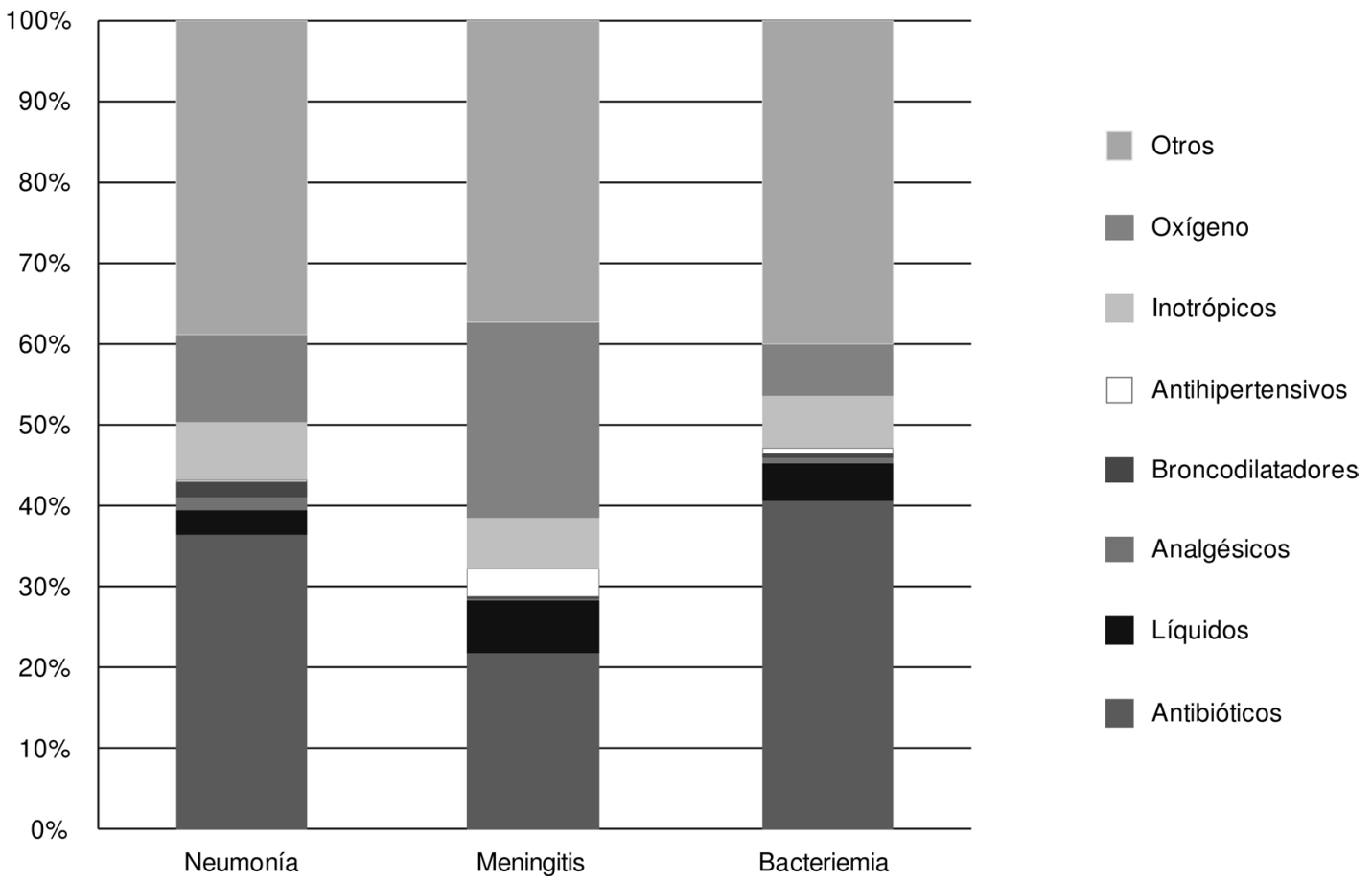

Figura 2. Distribución de costos directos de medicamentos por enfermedad (porcentaje).

en el costo total. Otro grupo de costos altos fue el clasificado como "otros", los cuales correspondieron a la heparina, los relajantes musculares y los sedantes, entre otros, así como el de las medidas de nutrición parenteral. El oxígeno, que debe administrarse de manera constante durante las estancias, representó $7 \%$ del costo total de medicamentos (figura 2).
La mortalidad general registrada en el estudio fue de $31,6 \%(n=19)$ para las tres enfermedades, presentándose $42 \%$ de los casos en los pacientes de más de 80 años. Analizando la mortalidad según la entidad, por neumonía fue de $21 \%$, por meningitis de $33 \%$ y por bacteriemia de $48 \%$. De las muertes en pacientes con neumonía $(n=7)$, $70 \%$ correspondió a hombres $(n=5)$ y $30 \%$ a 
mujeres $(n=2) ; 62 \%$ de los casos de mortalidad por neumonía se presentó en sujetos mayores de 65 años. Al analizar la mortalidad con respecto al perfil de riesgo, se encontró que la mayoría se produjo entre las personas de perfil moderado: $86 \%$ en pacientes con neumonía y $80 \%$ en pacientes con bacteriemia, en tanto que en el grupo de meningitis los casos se distribuyeron por igual en el perfil bajo y en el moderado. En el cuadro 1 se observa que hombres y mujeres representan igual número de casos de meningitis (50\%), y $83 \%$ de los casos se presentaron en el grupo de menor edad (18-44 años). Si se discriminan los casos que presentaron desenlace fatal, se observa que los sujetos fallecidos tuvieron un tiempo promedio de estancia de 11 días para neumonía, 3,6 días para meningitis y 4 días para bacteriemia.

\section{Costos asociados con el manejo ambulatorio de la neumonía adquirida en la comunidad}

La estimación de los casos de neumonía adquirida en la comunidad que presentó en el año 2006 el Ministerio de la Protección Social en Colombia mostró que de 6.000 neumonías al año, 1.500 casos se presentaron por enfermedad invasiva en personas mayores de 60 años (7). Los diagnósticos de neumonía atendidos por consulta externa se basan en la historia clínica y se complementan con estudios diagnósticos de rayos $\mathrm{X}$ de tórax, y pruebas de laboratorio como el hemograma. La decisión de manejo ambulatorio u hospitalario depende de los criterios de las guías del manejo establecido para la enfermedad grave y para la enfermedad cardiopulmonar asociada en personas mayores.

El tratamiento ambulatorio de la neumonía adquirida en la comunidad se hace de manera empírica porque no es común realizar pruebas de aislamiento microbiológico en la consulta externa. Streptococcus pneumoniae se ha identificado como el patógeno más común, pues suele representar hasta $35 \%$ de los casos de neumonía adquirida en la comunidad (8). El estudio de carga de enfermedad en adultos en Colombia estimó que S. pneumoniae estaba presente en $80 \%$ de las neumonías adquiridas en la comunidad (9).

Nuestro estudio presenta el cálculo de los costos totales de los recursos, expresados en pesos colombianos de 2010, de consulta externa o consulta de urgencias en casos que no requirieron hospitalización; la perspectiva utilizada para el análisis de costos correspondió a la de las EPS. Las tarifas consideradas como referencia se basaron en el manual tarifario del ISS para 2001 más 30 $\%$, debido a que en el momento tenían mayor representatividad en el mercado. Conscientes de la variabilidad en los precios de medicamentos, se trabajó con valores llevados a la unidad mínima (p/mg); el trabajo se realizó con las dosis diarias definidas por la OMS, aunque para este ejercicio en particular se incluyó la dosis recomendada por el grupo de expertos clínicos sin que la variabilidad haya sido grande. La base de este cálculo se encuentra en el artículo 1 del Decreto 4474 de 2010 y los precios que se incluyeron son los reportados por las bases SISPRO-SISMED del Ministerio de Protección Social. Estas tarifas fueron definidas a partir del análisis de los diferentes porcentajes de contratación a nivel nacional tanto para el sector público como para el privado.

Los costos directos asociados al manejo de la neumonía adquirida en la comunidad se cuantificaron para determinar las actividades y los insumos requeridos para el manejo ambulatorio de la neumonía adquirida en la comunidad tal como lo indican las guías de atención integral para la estimación de evaluación económica. Para el desarrollo de la estimación de costos de atención ambulatoria de la neumonía adquirida en la comunidad, se implementó la metodología Delphi de consenso (tres especialistas en neumología). Se obtuvo acuerdo entre los especialistas después de dos ciclos de consenso.

El costo de la consulta realizada por médico internista incluyó un control de seguimiento para la neumonía adquirida en la comunidad y se estimó en Col\$32.536 y contempló el costo de las ayudas diagnósticas como radiografía de tórax más un control radiológico para un total de Col\$53.716. Para los exámenes de laboratorio se tomó el hemograma a un costo de Col\$ 8.437 y el nitrógeno ureico más creatinina con un costo de Col\$11.485,50 en caso de enfermedad concomitante del paciente. Se consideró el costo de la tinción de Gram y el cultivo de esputo en Col\$35.470,50 y, en casos especiales, los gases arteriales en Col\$23.050.

Los lineamientos terapéuticos por consenso se basaron en las guías de manejo de la neumonía adquirida en la comunidad de la American Thoracic Society. Los costos de medicamentos incluyeron los valores promedio y ponderados tanto para genéricos como para moléculas originales ofrecidas en el mercado. El costo directo del tratamiento ambulatorio de antibiótico con macrólidos como 
claritromicina $(500 \mathrm{mg}$ cada 12 horas por 10 días) se calculó en Col\$15.663,12 y el grupo de cefalosporinas como segunda elección en este caso; el costo del tratamiento con cefuroxime en dosis de $500 \mathrm{mg}$ cada 12 horas por 10 días, se calculó en Col\$ 73.832,72. Para casos que cursaron con fiebre y dolor se calculó la formulación de un analgésico, en este caso, acetaminofén, a un costo de Col\$233,51 para un tratamiento de $500 \mathrm{mg}$ cada 8 horas durante 5 días. Se incluyó la utilización de un mucolítico del grupo del fluimucil, $200 \mathrm{mg}$ cada 12 horas por 2 días, con un costo de Col\$1.748. Se calculó que el costo total del manejo de la neumonía adquirida en la comunidad por consulta ambulatoria, con tratamiento inicial de claritromicina, se encuentra entre Col\$ 159.280 y un máximo de Col\$274.427 si se realiza tratamiento con cefuroxime y se anexa una toma de gases arteriales por cada paciente adulto.

\section{Discusión}

Este es el primer estudio de costos directos en neumonía, meningitis y bacteriemia por $S$. pneumoniae realizado en Colombia. La muestra de sujetos empleada en el estudio correspondió a personas afiliadas al Sistema General de Seguridad Social en Salud (SGSSS); $62 \%$ de los sujetos pertenecía al régimen contributivo y $38 \%$ al subsidiado.

En general, las variables que más incidieron en el costo total del manejo de las infecciones por $S$. pneumoniae fueron la estancia y los medicamentos, (antibióticos). Al analizar los costos directos totales para neumonía se encontró un costo promedio de Col\$ 12'178.949 (DE=Col\$ 14'264.174), del cual, $44 \%$ correspondió a medicamentos y $35 \%$ a estancia, constituyendo estos dos componentes el mayor rubro; $51 \%$ del costo promedio de la estancia (Col\$ 4'246.754, DE=Col\$ 5'954.456) correspondió a hospitalización en la unidad de cuidados intensivos y de éste, 58 \% correspondió a casos de neumonía clasificados en el grupo de perfil de riesgo moderado y relacionados con alguna enfermedad crónica concomitante; los grupos de edad predominantes se agruparon en el rango de 45 a 79 años. La mortalidad por neumonía se presentó en $37 \%$ de los casos y se asoció con presencia de enfermedades concomitantes; $86 \%$ de estos casos correspondieron a personas con perfil de riesgo moderado.

La variable del costo de medicamentos para la bacteriemia fue la más alta, representando 61 $\%$ del costo total, seguida por la neumonía con $44 \%$ y la meningitis con $23 \%$; estos porcentajes se correlacionaron con estancias de 10 y 15 días para el manejo de la infección con antibióticos intravenosos. Los antibióticos representaron el rubro más elevado en medicamentos, lo cual es de esperar para una infección bacteriana complicada (figura 2). En la mayoría de los casos el uso de antibióticos se ajustó a las guías de manejo de las instituciones y a los criterios de resistencia bacteriana. Los antibióticos representaron un alto porcentaje del costo de medicamentos, alcanzando $41 \%$ en el manejo de la bacteriemia en la unidad de cuidados intensivos. El costo promedio de los antibióticos para el tratamiento de la neumonía correspondió a $36 \%$ del total de costos y para el de la meningitis fue de $22 \%$. En este estudio se observó que, a diferencia de los resultados obtenidos en población infantil (10), los costos de neumonía por $S$. pneumoniae en adultos fueron superiores a los costos para el manejo de la meningitis. En cuanto a los costos por exámenes de laboratorio y ayudas diagnósticas el porcentaje se comportó de forma similar en las tres entidades.

Los costos analizados en este trabajo fueron superiores a los obtenidos en estudios latinoamericanos en los que se clasifica a Colombia como un país de bajo ingreso cuyos costos calculados fueron de US\$ 804, US\$1.030, y US\$ 1.053 para neumonía, meningitis y sepsis, respectivamente (11) (la tasa de cambio se calculó con la variación de cada año: 1 dólar $=$ Col $\$ 1.938$, variación promedio de 34 entre 2010 y 2011). Según estos datos, la información reportada por el estudio citado subestimó los costos en cerca de cuatro veces para neumonía, 2,5 para meningitis y tres veces para bacteriemia. Con estos datos, el costo real de las enfermedades causadas por $S$. pneumoniae resultaría considerablemente mayor al estimado (11).

La infección por $S$. pneumoniae sigue siendo una de las principales causas de neumonía adquirida en la comunidad y está asociada a una alta incidencia de mortalidad en adultos en Latinoamérica. Además, a medida que la expectativa de vida de la población aumenta, también aumentan las enfermedades concomitantes que elevan la vulnerabilidad a infecciones; por ello es necesario manejar correctamente la enfermedad desde su comienzo, ya que tiene un impacto importante en los costos del Sistema General de Seguridad Social en Salud de Colombia. Capacitar al personal de salud en el diagnóstico y manejo adecuado de la enfermedad, así como conocer la magnitud de los 
costos, puede ayudar a reducir costos y a motivar decisiones que reduzcan la carga de enfermedad atribuible a esta infección (5).

En las enfermedades producidas por $S$. pneumoniae los costos indirectos e intangibles pueden aumentar si se tiene en cuenta la asociación con discapacidad. Sabiendo que 58 $\%$ de los casos de neumonía por $S$. pneumoniae se presentan en personas menores de 58 años, es relevante tener en cuenta la carga de la enfermedad neumocócica, que agrupa neumonía, meningitis y bacteriemia, y que en Colombia se ha calculado en 2,03 AVAD por cada 1.000 habitantes adultos (9). De esta manera, el impacto de la infección por S. pneumoniae en el costo y la calidad de vida sería entonces significativo; así, pues, las medidas preventivas deberán estar encaminadas a manejar la infección a tiempo y evitar la hospitalización por complicaciones. A las empresas prestadoras de servicios de salud (EPS) les corresponde realizar programas de prevención en adultos mayores para evitar que las complicaciones de una infección prevenible no resulten en gastos crecientes para el SGSSS. La proporción de enfermedades crónicas se ha incrementado, por lo que los esfuerzos actuales deben orientarse a la prevención de infecciones por $S$. pneumoniae por medio de programas de prevención y control de enfermedades crónicas no transmisibles, así como a mejorar la efectividad de los programas de vacunación en adultos mayores.

La mayor limitante del estudio fue el número de sujetos incluidos en la muestra (60), hecho atribuible en parte a la exclusión de los casos de tratamiento de cáncer o con inmunosupresores. El estudio se hizo en sujetos hospitalizados y no se tomó en cuenta el costo ambulatorio de medicamentos y consultas de los pacientes dados de alta. Es posible que el costo no haya recibido un impacto por el margen correspondiente al tratamiento ambulatorio, que hubiera incrementado en algo el costo final por paciente.

El tratamiento ambulatorio de la neumonía adquirida en la comunidad seguirá siendo empírico si se tienen en cuenta las dificultades de obtención de una muestra adecuada para cultivo de $S$. pneumoniae en consulta externa o urgencias y el hecho de que los resultados del cultivo pueden tardar tres días o más, tiempo en que no se puede dejar al sujeto sin terapia con antibiótico, más aun sabiendo que en un poco más de la mitad de los casos no se logra aislar patógeno alguno (6). En Colombia, una neumonía ambulatoria con tratamiento idóneo y oportuno puede costar Col\$ 274.427 y un manejo tardío en personas mayores de 45 años puede incrementarlo en Col\$ 12'178.949. Las complicaciones de una infección por S. pneumoniae, como meningitis o bacteriemia, pueden incrementar los costos por paciente a Col\$ 7’533.187 y Col\$ 9'242.806, respectivamente. Un adecuado diagnóstico y tratamiento de la neumonía adquirida en la comunidad, así como la oferta de programas de vacunación para personas mayores de 45 años, pueden representar un ahorro importante de costos para el SGSS y una menor mortalidad debida a las tres entidades analizadas en el presente estudio.

\section{Agradecimientos}

Los autores agradecen a Carlos Arturo Torres Duque y Carlos Enrique Awad García, neumólogos de más de 20 años de experiencia, por la colaboración en el panel de expertos y en el desarrollo de la metodología Delphi en los dos ciclos del consenso, así como a María del Rosario Calderón por el diseño de la base de datos para la recolección de los costos y a Juan Manuel Reyes por el análisis estadístico de los datos en el programa Stata.

\section{Conflicto de intereses}

Los autores expresan que no existían conflictos de intereses al momento de la redacción del manuscrito.

\section{Financiación}

La investigación fue financiada por Pfizer Colombia.

\section{Referencias}

1. WHO. World Health Report 2005 - make every mother and child count. Fecha de consulta: 18 de enero de 2013. Disponible en: http://www.who.int/whr/2005/whr2005_en.pdf.

2. De Quadros CA. From global to regional: The importance of pneumococcal disease in Latin America. Vaccine. 2009; 27(Suppl.3):C29-32.http://dx.doi.org/10.1016/j.vaccine. 2009.06.006

3. Alvis N, De la Hoz F, Higuera AB, Pastor D, Di Fabio JL. Costos económicos de las neumonías en niños menores de 2 años de edad, en Colombia. Rev Panam Salud Pública. 2005;17:178-83. http://dx.doi.org/10.1590/\$102049892005000300005

4. Drummond M, O'Brien B, Stoddart G, Torrance G. Métodos para la evaluación económica de los programas de asistencia sanitaria. Segunda edición. Madrid: Ediciones Díaz de Santos; 2001.

5. Castañeda-Orjuela C, Alvis-Guzmán N, De la HozRestrepo F. Impacto de la enfermedad por Streptococcus pneumoniae en población adulta mayor en Bogotá, 
Colombia. Rev Salud Pública. 2010;12:38-50. http://dx.doi org/10.1590/S0124-00642010000100004

6. Sanguinetti CM, De Benedetto F, Donner CF. GP management of community-acquired pneumonia in Italy: the ISOCAP study. Monaldi Arch Chest Dis. 2005;63:23-9.

7. Reyes S, Martínez R, Vallés JM, Cases E, Menendez R. Determinants of hospital cost in community-acquired pneumonia. Eur Respir J. 2008;31:1061-7. http://dx.doi. org/10.1183/09031936.00083107

8. Isturiz RE, Luna CM, Ramirez J. Clinical and economic burden of pneumonia among adults in Latin America. Int $\mathrm{J}$ Infect Dis. 2010;14:e852-e6. http://dx.doi.org/10.1016/j.ijid. 2010.02.2262
9. Rosselli D, Rueda JD. Burden of pneumococcal infection in adults in Colombia. J Infect Public Health. 2012;5:354-9. http://dx.doi.org/10.1016/j.jiph. 2012.04.003

10. Constenla D. Evaluating the costs of pneumococcal disease in selected Latin American countries. Rev Panam Salud Pública. 2007;22:268-78. http://dx.doi.org/10.1590/ S1020-49892007000900007

11. Constenla D, de la Hoz F, O'Loughlin R, Sinha A, Valencia JE, Valenzuela MT. The burden of pneumococcal disease and cost-effectiveness of a pneumococcal vaccine in Latin America and the Caribbean: A review of the evidence and a preliminary economic analysis. Washington, D.C.: Sabin Vaccine Institute; 2009 